\title{
Developing a Coding Rubric for Students' Spatial Visualization Strategies
}

\section{Mrs. Adetoun Oludara Yeaman, Virginia Polytechnic Institute and State University}

Adetoun Yeaman is a second year PhD student in Engineering Education at Virginia Polytechnic Institute and State University (Virginia Tech). She received here MS in Mechanical and Nuclear Engineering and her BS in Biomedical Engineering both from Virginia Commonwealth University. She is currently a Graduate Research Assistant. In her first year, she taught two semesters of a freshman engineering course series, Foundations of Engineering. Her research interests include multimedia learning, design education and empathic design.

Address: Virginia Tech Engineering Education (MC 0218) 345 Goodwin Hall, 635 Prices Fork Rd, Blacksburg, VA 24061.

\section{Dr. Diana Bairaktarova, Virginia Tech}

Diana Bairaktarova is an Assistant Professor in the Department of Engineering Education at Virginia Tech and the Director of the Abilities, Creativity, and Ethics in Design [ACE(D)] Lab.

\section{Prof. Tamara Knott, Virginia Tech}

Tamara Knott is Associate Professor of Engineering Education at Virginia Tech. Her primary responsibility centers on teaching in the First Year Program. Her interests include assessment and pedagogy. Within ASEE, she is a member of the First-year Programs Division, the Women in Engineering Division, the Educational Research and Methods Division, and the Design in Engineering Education Division. She is also a member of the Society of Women Engineers (SWE) and is the Faculty Adviser for SWE at VT. 


\title{
Developing a Coding Rubric for Students Visualization Strategies
}

\begin{abstract}
There are many calls for increasing the number and diversity of students pursuing STEM careers. Equally important is retaining those students who initially express interest in these careers. One of the myriad of factors that influences students' success in a STEM major is their spatial visualization ability. Fortunately, research has shown that spatial ability can be developed through practice and training. This possibility highlights the usefulness of strategies that can be used for solving spatial tasks. There are several reliable spatial ability measures including paper cutting tests, mental rotation tests, and cross section tests. The current study examines strategies that students reported using while taking a cross section test, Santa Barbara Solid Test (SBST) and this complete research paper describes the development of a coding rubric based on these strategies.
\end{abstract}

Keywords: spatial visualization, spatial abilities, first-year engineering, Santa Barbara Solid Test, cross-sections, spatial strategies

\section{Background}

Spatial visualization ability is critical for success in STEM disciplines. For example, in medicine, learning to read medical images requires the ability to understand cross-sections [1]. Wanzel, Hamstra, Anastakis, Matsumoto, \& Cusimano, [2] also reported a correlation between medical students' scores in mental rotation and their performance on a surgical procedure, Z-plasty. In engineering, higher abilities in cross-sectioning have been linked to better performance in Mechanics of Materials courses [3]. Two categories of spatial reasoning, as defined by Linn and Petersen [4], are mental rotation and spatial visualization. Mental rotation involves the ability to manipulate three-dimensional (3D) objects in one's mind by rotation, and spatial visualization involves the ability to manipulate three-dimensional (3D) objects in one's mind through mental cutting and unfolding. A number of tests are routinely used to assess spatial skills. These include Vandenberg-Kuse's Mental Rotation Test (MRT) and the Purdue Spatial Visualization Test: Rotations (PSVT: R) for mental rotation skills and the Mental Cutting Test (MCT) and Santa Barbara Solids Test (SBST) for mental cutting skills.

Of value for the recruitment and retention of STEM students is the fact that numerous studies have demonstrated the ability to improve spatial ability through training. A meta-analysis of training studies by Uttal et al. [5] concludes that spatial skills are malleable and that spatial training programs may play an important role in the education of STEM professionals. Numerous engineering programs working with the ENGAGE Engineering Project [6] offer courses in spatial visualization. These courses have been shown to positively impact participant spatial skills and retention in engineering programs [7], [8], [9]. A number of studies have also investigated the role of solution strategy in spatial performance. In 1991, Schultz [10] developed and tested the Spatial Strategy Questionnaire (SSQ) and used it to examine the contribution of strategy to variations in spatial performance on a number of spatial tasks including mental rotation. This study found a significant contribution of strategy to performance in mental rotation higher performance on the MRT being associated with the use of a strategy involving 
"moving the object" and avoidance of a strategy of the use of "key features". In a study of the influence of design training and spatial solution strategies on spatial ability, Lin [11] reported that research participants who used "holistic" solution strategies, which involve rotating objects, performed better on the MRT than those who used "analytic" solution strategies, such as "counting". In the development and testing of the SBST as an instrument for measuring cognitive ability, Cohen and Hegarty [12], noted that participants could use analytic strategies related to "key features" to eliminate answer choices in some problems. Their results also support the use of strategies associated with "holistic" solution strategies involving moving the object or moving relative to the object by those with higher spatial ability. In our study, we build on the research studies that highlight the importance of spatial skills development in STEM fields and reveal that students can be trained in these skills. The aim of our study is to investigate self-reported solution strategies for a particular spatial visualization test, SBST, as a step towards training students in effective spatial visualization strategies. We argue that in order to present useful interventions to train students in developing spatial visualization skills, we should first understand how students approach spatial visualization tasks.

\section{The Present Study}

\section{Methods}

This complete research paper describes the development of a coding rubric based on strategies for tackling spatial reasoning tasks as identified by engineering students. We take a qualitative approach to explore how students go about tackling spatial reasoning tasks. Our study is phenomenologically informed as this strategy is the most appropriate of the common qualitative traditions (phenomenology, grounded theory, narrative research, ethnography and case study) [13], [14] for our study. We have used a phenomenologically informed strategy rather than phenomenology itself as we have adopted the philosophies of phenomenology in guiding our study our practice [13]. This involves clearly articulating the phenomenon under study, allowing the experience of the participants to drive the development of descriptions of the essences of the experience being examined and avoiding any presuppositions on the part of the researcher [13]. In our study, we explore experiences of freshmen engineering students in a remedial spatial visualization course while taking a spatial reasoning test, the SBST. Through this study, we established a coding rubric by examining strategies that students reported using in response to an open-ended question. The SBST is 30-item multiple choice psychometric test that was developed to measure individual differences in a spatial visualization task. It involves identifying the cross section resulting from the intersection of a cutting plane and a geometric solid [12]. A sample problem on the test is shown in Figure 1 below. 


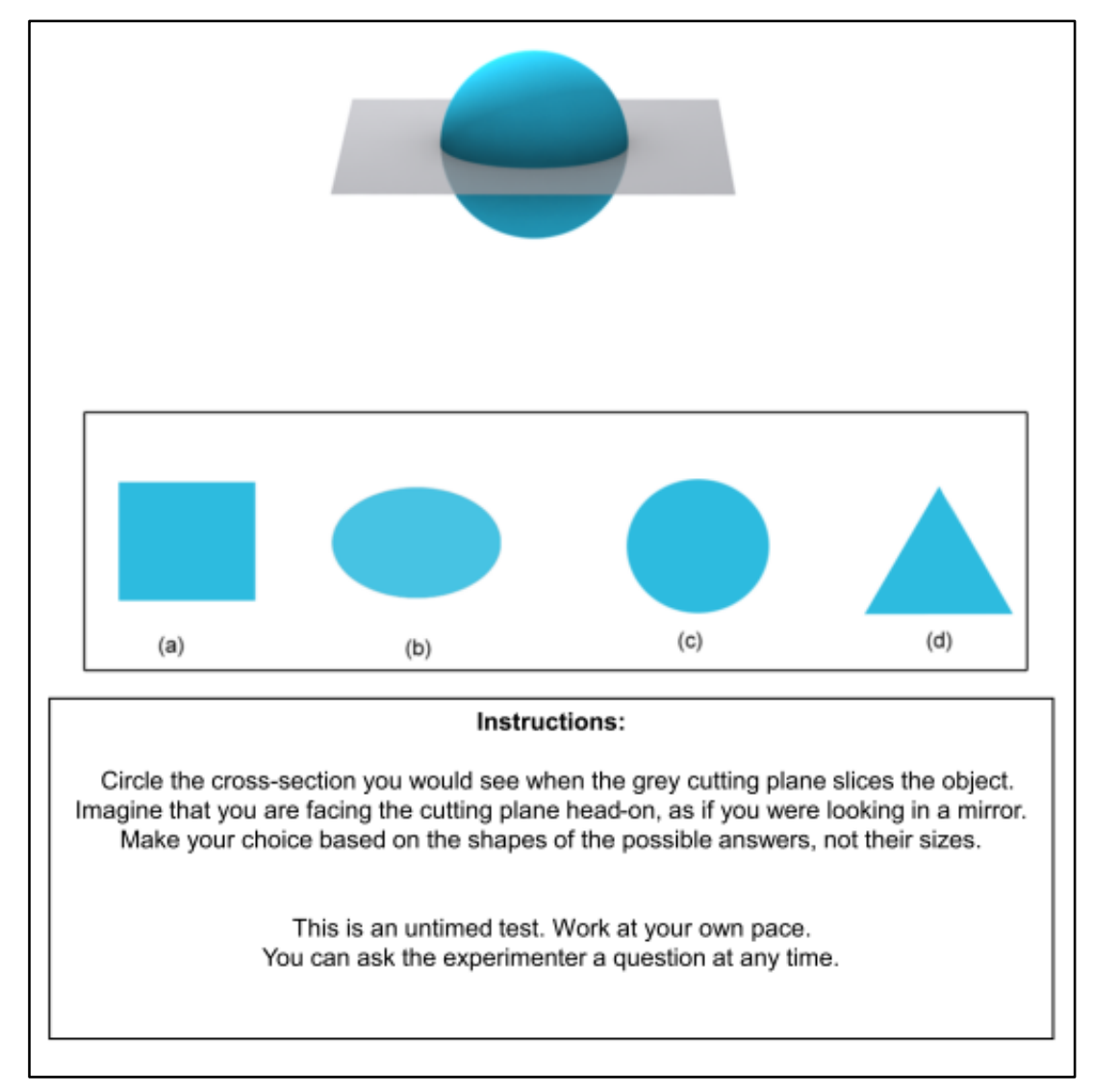

Figure 1: SBST sample problem [15]

\section{Participants and Sampling}

The participants in our study were purposefully sampled. They are first-year engineering (FYE) students, enrolled in an Introduction to Spatial Visualization course at a large land grant University. This course is recommended for first-year students who score below the threshold of $60 \%$ correct on the Purdue Spatial Visualization Test: Rotation (PSVT:R) on entry into the engineering program. The goal of the course is to help students improve their spatial visualization skills. The sample for our study consists of 183 students. At the beginning of the semester, students completed the SBST in class electronically. At the end of the test, students were asked to share any strategies they have used while taking the 30 questions of SBST. The specific prompt given to students is as follows: "Did you have a strategy for choosing the answer to each problem? If so, can you explain your strategy in a few words?" In addition, student reported demographic information had been collected at the beginning of the semester. Among the 169 students who provided their demographic information, 140 responded to the prompt at the end of the SBST. This is the sample of 140 students whose responses were considered for analysis in this this study. The responses to the open-ended question were collected and analyzed first by looking for emerging categories. The results included evidence of differences in the strategies students used on the test. 


\section{Analysis}

One researcher did initial coding of participants' responses. The researcher used open coding which involves getting familiar with data, categorizing data into broad groups and coming up with interpretations [16], [17]. Ten themes emerged from this initial coding process, some of which were in-vivo codes [18] from participants' responses. The ten themes and the data set were sent to two other researchers. The two researchers independently coded students' responses and then the coding team met together to discuss the emerging categories and the initial coding rubric. Inter-coder agreement was established between the three coders and six categories of strategies were finalized, namely mental action, guessing, guiding rule, intuition, process of elimination, and thinking. Students whose responses were incorporated into the report were given pseudonyms, which are used for the discussion of results below.

\section{Results}

The six themes that emerged from the coding of participants' responses are shown in italics in Table 1 below: mental action, guessing, guiding rule, intuition, process of elimination and thinking. The responses of participants who reported not using any strategies were classified as No strategy as shown in Table 1. These responses include: "no", "not really", "no strategy", "little to no strategy", "no i did not have a strategy" and "n/a". Additionally, responses of participants that fit into a combination of multiple strategies are presented as Combination

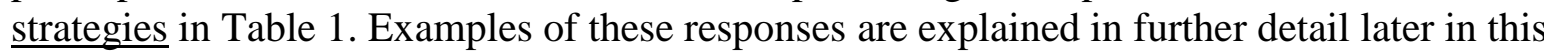
section.

Table 1: Summary of responses

\begin{tabular}{|l|l|}
\hline Reported Strategy (if any) & Number of participants \\
\hline No strategy & 35 \\
\hline Mental action & 73 \\
\hline Guessing & 1 \\
\hline Guiding rule & 11 \\
\hline Intuition & 4 \\
\hline Process of elimination & 3 \\
\hline Thinking & 4 \\
\hline Combination of strategies & 9 \\
\hline Overall responses & $\mathbf{1 4 0}$ \\
\hline
\end{tabular}


Mental action. This strategy implies that the student visualized, imagined, created a mental picture or did some level of mental manipulation of the object to get the answer. While most of the mental processes students mentioned were exclusively happening in the mind, others were uniquely different, for example drawing in air. In the case of John, his response reflects some sort of imagination or visualization (drawing from his own words), "I tried to visualize the parts being moved away from each other and what they would look like after they had moved apart." Cindy on the other hand, took a more physical approach as indicated in her response, "My strategy for choosing the answer to each problem was drawing it out in the air with my hand and trying to imagine myself looking down on it in order to figure it out." Her approach involved using a physical process of drawing in the air to help her imagine what she expected to see in her answer choice.

There are several other responses that reflect some sort of invisible action taking place in the mind as highlighted in the following examples. Kim indicated using her imagination, " $M y$ strategy for choosing the answer to each problem was to visualize that I was a mirror, and the grey section was what I was seeing, and I tried to imagine the view I had of the object." Her approach is similar to Cindy's in the aspect of imagining viewing the object. It is also somewhat similar to John's in the sense that she was also visualizing a movement however; in contrast to John's, Kim is the one moving and not the parts. She imagined herself as a mirror seeing the grey section of the object. Byron was another student who reported visualizing, "Checking the initial slant of the cut and matching then visualizing what the interior would look like in 2D". Finally, Jin indicates imagined movements such as cutting and rotating towards her, "i'll (sic) cut the image in my head and just rotate it towards me so the face of the object is "looking" towards $m e$ ". These movements point to an application of the strategy that has been categorized as mental action. It was interesting to see that some students did not think they used any strategies but still gave responses that indicated strategies. For instance, Tanner said, "no I just try to see the shape the best I can". This response implies some level of mental action taking place. In the process of closely examining the object, he is likely able to imagine what the right cross section should look like. Consequently, Tanner's response was coded as mental action.

Guessing. Responses that used the word "guess" or explained that the student arrived at a conclusion by chance or without showing evidence of deliberate reasoning were classified as guessing. For instance, Mia responded with, "No, I just guessed on each question." In this case, she specifically had "guess" in her response. The other students' who responses involved guessing also reported using other strategies. These are further discussed in the section on combined strategies below.

Guiding rule. Guiding rule implies that in the participant responses, the student(s) used a standard or criteria to judge which option is likely to be the answer for example, student responses that involved the use of if-then logic ("if...then...") or stating a specific criterion that led to the answer ("whatever is...is the answer"). Jason applied an if-then logic as shown in his response, "If the plane intersecting the shape is slanted then the cross section will look weird like it is stretched out. If the plane is straight, then it will look normal." Similarly, Ming's response conveys an if-then approach as well: "If it is cut diagonally, and the shape does not have parallel sides than (sic) the resulting cross-section will have shapes that do not have 
parallel sides." Conversely, Jessica had a rule based on similarities her saw between the original image and an option within the multiple choices, "I tried looking at how the image was and observed the prospective (sic) of each. If I found an answer that had a similar perspective as what the image above had I typically went with that choice." She explicitly states a criterion that she used as the basis for choosing her answers. An interesting response came from Penina, "I realized halfway through that I just needed to check which answer would slide into the separator/line". According to her response it appears that she initially tried to answer the test questions without using any strategy but halfway into the process, decided on one. Her strategy involved a rule that guided her answer choices, checking which answer would slide into the separator line.

Intuition. In some students' responses, the word intuition was used in the response or the response showed that the student came to an understanding of the answer immediately without the need of conscious reasoning. Two participants, Dave and Phat specifically used the word, "Intuition" to describe their strategy. Sarah also indicated applying intuition saying, "I just looked at it”. From simply looking at the object, she chose her answer.

Process of elimination. In the responses categorized as process of elimination, the participants recognized which options were not likely to be the answer and narrowed their options by setting the unlikely choices aside. For example, Kai gave the following response, "my strategy is just to compare the picture with the answer and find the things are not true". Comparing the original object with the answer choices, he was able to eliminate answers that were not true to arrive at his answer choice. Mike applied a similar strategy as well, "I kind of compared(sic) the answer choices back to the object to eliminate some." Only a few students applied the process of elimination as their strategy.

Thinking. In the thinking category, student responses involved an expression of some level of thinking, typically by using the word thinking, thought or think explicitly. Although we understand that some level of thinking may occur before most students decide on an answer choice, this category is unique in the sense that it is based on students' use of words that imply thinking only. In contrast, mental action is more explanatory as some participants described what was going on in their minds other than simply thinking. Brittany gave a brief description of her strategy as, "Think. It through step by step (sic)". Her approach involved thinking step-by-step and her response was categorized as thinking because she used the word "think". Likewise, Mike had a similar response, short and with a description that involved a derivative of the word think, "Just thinking about it........" Brittany and Mike are two of the few participants whose strategies could be categorized as thinking. Other students applied thinking, but their categories also fit into another category and are described in the combined strategies section.

Combined strategies. Responses were categorized as combined strategies if they appeared to have elements of more than one strategy or where the participant specifically reported using two different strategies. For instance, Brooke's response indicated elements of two different strategies, "I look at what shape the outside half looks like on the screen, and try to think about what that piece looks like on the other side if I removed the back half." This response fits into two categories, thinking and mental action. Similarly, Jack also indicated elements of two categories of strategies, guessing and intuition. He said, "I just picked what I thought was best 
and tried not to over think the questions". This is a more indirect response showing that he did not give much thought to his response. This can be categorized as him guessing, in the sense that he simply chose an answer without really putting much thought into it. It could also have an element of intuition because he could quickly get a sense of the correct answer therefore not needing to put much thought into the process. However, he did mention that he picked what he "thought" was best indicating that some degree of thinking was involved in his decision making.

A combination of strategies is also seen in Zachary's response, "Yes. I just look at what points of the cutting plane touch the object." Although he does not mention what happens after he looks for the specific points, his response captures elements of both mental action and a guiding rule. The mental action aspect is demonstrated in how he looks at the object for something specific. He also signals to the use of a guiding rule by indicating what he was looking for, what points of the cutting plane touch the object. This same combination of elements of mental action and guiding rule is captured in Nate's response, "Look to see if it is at an angle in the picture. Try to visualize in my head. " Similar to Zachary, he looks for something specifically. However, he was more explicit about his use of a strategy that fits into the mental action category, he tried to visualize in his head. This aspect of visualizing is also echoed by Ethan, "Reason and process of elimination. I also attempted to visualize the cuts. " In addition to attempting to visualize the cuts and reasoning, he explicitly mentions that he applied the process of elimination. His response exemplifies a combination strategy involving mental action and process of elimination.

Lily's response indicated both guiding rule and mental action, "I tried to pick the shape that most closely resembled the two individual shapes before combining them into one object". The phrase, "that most closely resembled" in her response is indicative of a specific outcome that she was hoping to see, serving as a sort of guidance for her answer choice. This strategy points to the use of a guiding rule, in the sense that she bases her answer on a particular criterion. Taking into account the overall approach she takes points to mental action as well. She picks a shape, which would likely involve examining the objects in the answer choices as well as the shapes that would emerge from slicing the original object. Then she combines the shapes; this does not mean she literally takes the shapes and combines them with her hands, rather it has to happen in her mind.

Similar to Tanner, who was mentioned earlier, another student, Javier also indicated that he used no strategy but then provided an additional statement; Javier's additional statement, "No, just go with the flow.", suggests the use of two strategies, guessing and intuition. The statement suggests he simply guessed the answers or gave his answer choices based on intuition in the sense that he was able to get a sense of the right answer with little to no effort on his part. His response was categorized as a combination strategy, as it connotes elements of intuition and guessing.

\section{Discussion}

In this study, we build on the research studies that highlight the importance of spatial skills development in STEM fields and reveal that students can be trained in these skills. The aim of our study was to investigate self-reported solution strategies for a particular spatial visualization test, SBST, as a step towards training students in effective spatial visualization strategies. We argued that to present useful interventions to train students in developing spatial visualization 
skills, we should first understand how students approach spatial visualization tasks.

We have examined strategies that students reported using while taking a cross section test, the SBST. In the process of developing a coding rubric based on these strategies, six themes have emerged - mental action, guessing, guiding rule, intuition, process of elimination, and thinking. Our findings support prior research on the role of solution strategy in spatial performance [10], [11] and extends the existing body of literature by confirming the use of strategies in variations in spatial performance on spatial tasks including mental cutting. While we did not investigate the contribution of strategy to performance in mental cutting, the themes in our coding rubric confirm Cohen and Hegarty's [12] study that people can use analytic strategies related to "key features" to eliminate answer choices in some problems - guiding rule and process of elimination. Our results also support the use of strategies associated with "holistic" solution strategies involving moving the object or moving relative to the object by those with lower spatial ability - mental action.

The results of our study can be useful in understanding how students tackle spatial tasks. This can provide some insight on how to offer more efficiently spatial visualization training, particularly cross section related spatial problems. Our next steps are to investigate if gender influences one's strategies in solving cross-sectional problems and to further refine the coding rubric using a second sample of the same population (next cohort of FYE students). We also plan to examine strategies students use in spatial ability tests other than cross-section test. It was difficult to fit some strategies into one specific category, for example Jack's response hinted at elements of thinking, intuition and guessing. It is interesting to note that one can make a choice among options and guess correctly through intuition. This makes the line between defining what intuition is and what is considered guessing challenging.

\section{Limitations and Future Studies}

This study is a first attempt at understanding students' strategies. Categories can be refined with more understanding from future studies. All strategies are related to one test, the SBST, which is focused on one subskill (mental cutting) among other spatial visualization subskills (e.g. rotation, imagery, etc.). The analysis in this study requires a considerable level of interpretation on the part of the researchers as we may not know exactly what the students mean by their responses since we did not have the opportunity to follow up with them and clarify their own meanings. In future studies, we will investigate if similar or different strategies are used in solving other spatial visualization tests and spatially-related problems.

\section{References}

[1] C. A. Cohen and M. Hegarty, "Sources of Difficulty in Imagining Cross Sections of 3D Objects," in 29th Annual Conference of the Cognitive Science Society (CogSci 2007), 2007, pp. 179-184.

[2] K. R. Wanzel, S. J. Hamstra, D. J. Anastakis, E. D. Matsumoto, and M. D. Cusimano, "Effect of visual-spatial ability on learning of spatially-complex surgical skills,” Lancet, vol. 359, no. 9302, pp. 230-231, 2002. 
[3] O. Ha and S. A. Brown, "Spatial Reasoning Difference between Civil and Mechanical Engineering Students in Learning Mechanics of Materials Course: A Case of Cross-sectional Inference," in ASEE Annual Conference \& Exposition, 2017.

[4] M. C. Linn and A. C. Petersen, "Emergence and Characterization of Sex Differences in Spatial Ability: A Meta-Analysis," Child Dev., vol. 56, no. 6, pp. 1479-1498, 1985.

[5] [1] D. H. Uttal et al., "The Malleability of Spatial Skills: A Meta-Analysis of Training Studies," Psychol. Bull., vol. 139, no. 2, pp. 352-402, 2013.

[6] N. L. Veurink et al., "Enhancing visualization skills-improving options and success (EnViSIONS) of engineering and technology students," Eng. Des. Graph. J., vol. 73, no. 2, pp. 1-17, 2009.

[7] N. Boersma, A. Hamlin, and S. Sorby, "Work in progress - impact of a remedial 3d visualization course on student performance and retention," in 34th Annual Frontiers in Education (FIE), 2004, pp. 600-601.

[8] N. L. Veurink and S. A. Sorby, "Raising the bar? Longitudinal study to determine which students would most benefit from spatial training," in American Society for Engineering Education, 2011.

[9] A. Srivasavan, J. Smith, and D. Bairaktarova, "Identifying Freehand Sectional View Technical Drawing Activities in Engineering Design Graphics Course to Enhance Spatial Skills of Engineering Students," in Proceedings of the ASME 2016 International Design Engineering Technical Conferences \& Computers and Information in Engineering Conference IDETC/CIE, 2016.

[10] K. Schultz, "The Contribution of Solution Strategy to Spatial Performance," Can. J. Psychol., vol. 45, no. 4, pp. 474-491, 1991.

[11] H. Lin, "Influence of design training and spatial solution strategies on spatial ability performance," Int. J. Technol. Des. Educ., vol. 26, no. 1, pp. 123-131, 2016.

[12] C. A. Cohen and M. Hegarty, "Inferring cross sections of 3D objects: A new spatial thinking test," Learn. Individ. Differ., vol. 22, no. 6, pp. 868-874, 2012.

[13] J. W. Creswell, Qualitative Inquiry and Research Design: Choosing Among Five Approaches, 3rd ed. Thousand Oaks, California: Sage Publications, Inc., 2013.

[14] J. W. Creswell, Research Design: Qualitative, Quantitative and Mixed Methods Approaches, 4th ed. Los Angeles: Sage Publications, Inc., 2014.

[15] C. A. Cohen, "Spatial Intelligence Learning Center (SILC)," 2015. [Online]. Available: http://www.spatiallearning.org/. 
[16] M. Borrego, "Conceptual Difficulties Experienced by Trained Engineers Learning Educational Research Methods,” J. Eng. Educ., vol. 96, no. 6, pp. 91-102, 2007.

[17] J. W. Creswell, "Five Qualitative Traditions of Inquiry," in Qualitative inquiry and research design: choosing among five traditions, London: Sage Publications, 1998, pp. 193-212.

[18] J. Saldana, The Coding Manual for Qualitative Researchers, 3rd ed. Los Angeles: Sage, 2016. 